\title{
Neutrino mass from bremsstrahlung endpoint in coherent scattering on nuclei
}

\author{
Alexander Millar, ${ }^{1,2, *}$ Georg Raffelt, ${ }^{3, \dagger}$ Leo Stodolsky, ${ }^{3, \$}$ and Edoardo Vitagliano, ${ }^{3, \S}$ \\ ${ }^{1}$ The Oskar Klein Centre for Cosmoparticle Physics, Department of Physics, \\ Stockholm University, AlbaNova, 10691 Stockholm, Sweden \\ ${ }^{2}$ Nordita, KTH Royal Institute of Technology and Stockholm University, \\ Roslagstullsbacken 23, 10691 Stockholm, Sweden \\ ${ }^{3}$ Max-Planck-Institut für Physik (Werner-Heisenberg-Institut), \\ Föhringer Ring 6, 80805 München, Germany
}

(Received 18 October 2018; published 10 December 2018)

\begin{abstract}
We calculate the coherent bremsstrahlung process $\nu+\mathcal{N} \rightarrow \mathcal{N}+\nu+\gamma$ off a nucleus $\mathcal{N}$ with the aim of revealing the neutrino mass via the photon endpoint spectrum. Unfortunately, the large required power of a monochromatic neutrino source and/or large detector mass make it difficult to compete with traditional electron-spectrum endpoint measurements in nuclear $\beta$ decay. Our neutral-current process distinguishes between Dirac and Majorana neutrinos, but the change of the photon spectrum is of the order of $m_{\nu} / E_{\nu}$ and thus very small, despite the final-state neutrino coming to rest at the photon endpoint. So the "Dirac-Majorana confusion theorem" remains intact even if $E_{\nu} \gg m_{\nu}$ applies only for the initial state.
\end{abstract}

DOI: 10.1103/PhysRevD.98.123006

\section{INTRODUCTION}

Two important questions of neutrino physics could be addressed if it were possible to have an experimental source of "stopping" or "slow" neutrinos. The first is that of the neutrino mass. If the neutrino has an energy comparable to its mass, then various phase space or kinematic factors will vary in a way involving the mass and so offer a way of determining it.

Secondly, there is the question of whether neutrinos are Majorana or Dirac. If the neutrino is a self-conjugate particle (Majorana) it has only an axial current, as opposed to the non-self-conjugate (Dirac) case where it has both vector and axial currents. According to the "confusion theorem" [1], however, both cases lead to the same experimental results when the neutrino is relativistic. But with slow or nonrelativistic neutrinos, differences can appear in principle and can offer a way to determine the nature of the neutrino.

Unfortunately neutrinos are invariably produced with relativistic energies so that it has been difficult to carry out

\footnotetext{
*alexander.millar@fysik.su.se

raffelt@mpp.mpg.de

les@mpp.mpg.de

sedovita@mpp.mpg.de
}

Published by the American Physical Society under the terms of the Creative Commons Attribution 4.0 International license. Further distribution of this work must maintain attribution to the author(s) and the published article's title, journal citation, and DOI. Funded by SCOAP ${ }^{3}$. such studies. One line of work, such as the KATRIN experiment [2], has been to study $\beta$-decay near the endpoint of the spectrum where the electron has almost all the energy so that the neutrino is slow. This approach involves very sensitive and elaborate electron spectrometry. Furthermore, as this is a charged-current process, it is not sensitive to the Majorana vs Dirac question.

A new signature to search for low-mass weakly interacting massive particle (WIMP) dark matter was recently proposed [3] in the form of coherent bremsstrahlung $\chi+\mathcal{N} \rightarrow \mathcal{N}+\chi+\gamma$. The photon is emitted by the very nonrelativistic nucleus $\mathcal{N}$ with a mass taken to be much larger than that of the WIMP. At the endpoint of the photon spectrum, all kinetic energy of the WIMP is released as electromagnetic energy which is relatively easy to detect with good precision. So in the CM frame, which here is almost the same as the laboratory frame, both the nucleus and WIMP come fully to rest when the photon energy is maximal. The crucial point is that the photon spectrum, although at a low absolute level, continues smoothly all the way to the maximum possible energy.

Inspired by this idea and by the recent observation of coherent neutrino scattering [4] one may wonder if the same process, substituting a neutrino $\nu$ for the WIMP $\chi$, could be useful to measure the neutrino mass $m_{\nu}$ because, near the photon endpoint spectrum, one produces slow neutrinos. For a fixed initial energy $E_{\nu}$, the photon endpoint energy and the shape of the endpoint spectrum must depend on $m_{\nu}$. A related idea is to produce the neutrinos themselves through an inelastic process [5]. 
The purpose of this short paper is to calculate the bremsstrahlung spectrum of this process, which is coherent both for the neutrino-nucleus and nucleus-photon interaction, with particular attention to the Dirac vs Majorana difference. Moreover, we will estimate the detection rate for a plausible experimental setup.

\section{CALCULATION OF NEUTRINO INDUCED BREMSSTRAHLUNG}

We consider a monochromatic neutrino beam scattering off a heavy and stable nucleus, such as tungsten. The elastic version of this process, i.e., without photon emission, was calculated long ago [6]; however due to the relativistic nature of the outgoing neutrino this process is insensitive to the neutrino mass. The same cannot be said for the bremsstrahlung process shown in Fig. 1 if we consider the regime where almost all of the available kinetic energy is taken by the photon. We will neglect the effects of the nucleus being inside an atom because the timescale of the interaction is much faster than the average timescale for the electrons to respond [3].

The bremsstrahlung cross section follows from the Feynman diagrams shown in Fig. 1, where we denote the neutrino initial and final four-momenta $p$ and $p^{\prime}$, the nucleus four-momenta $q$ and $q^{\prime}$, and the photon fourmomentum $k$. The calculation vastly simplifies in our case where the nuclear mass $M$ is much larger than the neutrino energy $E_{\nu}$ or the photon energy $\omega$, allowing us to ignore recoil effects. Moreover, in this limit the nucleus propagator simply contributes $(M \omega)^{-2}$ to the squared matrix element. The photon-nucleus vertex contributes a factor $\left|\epsilon_{\mu} \Delta q^{\mu}\right|^{2}$, where $\epsilon$ is the photon polarization vector and $\Delta q=q-q^{\prime}$ the four-momentum change of the charged particle. The photon transversality condition $\epsilon_{\mu} k^{\mu}=0$ implies that we can add or subtract $k$ to $\left(q-q^{\prime}\right)$ so that, after imposing the energy-momentum conservation of the overall process, $\epsilon_{\mu} \Delta q^{\mu}=-\epsilon_{\mu} \Delta p^{\mu}$. In the Coulomb gauge $\epsilon$ has only spatial parts and after averaging over directions of photon emission and summing over photon polarizations, the rate is proportional to $|\Delta \mathbf{p}|^{2}$. Overall we find the bremsstrahlung cross section
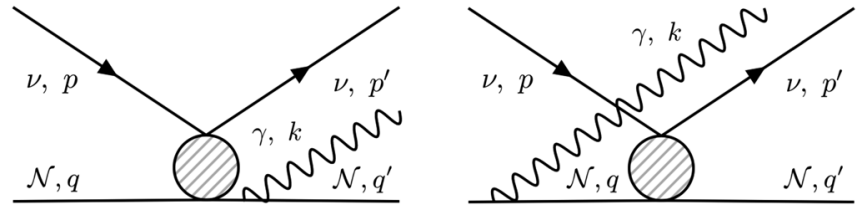

FIG. 1. Feynman diagrams for coherent neutrino-nucleus scattering with bremsstrahlung. The neutrino is denoted $\nu$ with initial and final momenta $p$ and $p^{\prime}$, the nucleus $\mathcal{N}$ with $q$ and $q^{\prime}$, and photon $\gamma$ with momentum $k$.

$$
\begin{aligned}
\frac{d^{4} \sigma_{\mathrm{b}}}{d^{3} \mathbf{p}^{\prime} d \omega}= & \frac{Z^{2} \alpha}{(2 \pi)^{3}} \frac{1}{12 M^{4} E_{\nu}^{\prime}|\mathbf{p}|} \frac{|\Delta \mathbf{p}|^{2}}{\omega}\left|\mathcal{M}_{\mathrm{s}}\right|^{2} \\
& \times \delta^{(0)}\left(E_{\nu}-E_{\nu}^{\prime}-\omega\right),
\end{aligned}
$$

where $\mathcal{M}_{\mathrm{s}}$ is the matrix element of $\nu \mathcal{N}$ scattering in the large- $M$ limit.

We then integrate over the remaining phase space, where we use $d^{3}\left|\mathbf{p}^{\prime}\right|=2 \pi d \cos \theta\left|\mathbf{p}^{\prime}\right|^{2} d\left|\mathbf{p}^{\prime}\right|$. This is used to integrate over the energy delta function, so a Jacobean $d\left|\mathbf{p}^{\prime}\right|=E_{\nu}^{\prime} /\left|\mathbf{p}^{\prime}\right| d E_{\nu}^{\prime}$ comes in and we find

$$
\frac{d^{2} \sigma_{\mathrm{b}}}{d \omega d \cos \theta}=\frac{2 Z^{2} \alpha}{3 \pi \omega} \frac{|\Delta \mathbf{p}|^{2}}{M^{2}} \times \frac{1}{2 \pi} \frac{1}{16 M^{2}} \frac{\left|\mathbf{p}^{\prime}\right|}{|\mathbf{p}|}\left|\mathcal{M}_{\mathrm{s}}\right|^{2},
$$

where $|\mathbf{p}|=\sqrt{E_{\nu}^{2}-m_{\nu}^{2}}$ and $\left|\mathbf{p}^{\prime}\right|=\sqrt{\left(E_{\nu}-\omega\right)^{2}-m_{\nu}^{2}}$. Moreover, $\theta$ is the neutrino scattering angle and the second factor is the $\nu \mathcal{N}$ scattering cross section $d \sigma_{\mathrm{s}} / d \cos \theta$, however with the alteration that $E_{\nu}^{\prime}=E_{\nu}-\omega$ (heavy nucleus approximation).

In the soft-photon limit $\left(\omega \ll E_{\nu}\right)$ this becomes the ordinary elastic $\nu \mathcal{N}$ scattering cross section. In this limit we can ignore $\mathbf{k}$ in the momentum balance so that $|\Delta \mathbf{p}|=$ $|\Delta \mathbf{q}|$ and therefore $|\Delta \mathbf{p}|^{2} / M^{2} \rightarrow|\Delta \mathbf{v}|^{2}$, where $\Delta \mathbf{v}$ is the velocity change of the nucleus. Our result then agrees with the usual classical nonrelativistic soft-photon bremsstrahlung rate, serving as a verification of our overall factors.

Finally, we need the matrix element $\left|\mathcal{M}_{\mathrm{s}}\right|^{2}$ derived in the Appendix. Here we assume a Dirac neutrino, discussing the differences from a Majorana neutrino in Sec. III. We assume that the nucleus is scalar; however our results hold for any large nucleus, such as tungsten, as only the coherently enhanced vector coupling matters [6]. In addition, we use $M \gg E_{\nu} \gg m_{\nu}$, ignore the recoil of the nucleus, and ignore $m_{\nu}$ everywhere except in the finalstate neutrino variables. With these approximations we find

$$
\begin{aligned}
\left|\mathcal{M}_{\mathrm{s}}\right|^{2}= & 4 G_{\mathrm{F}}^{2} M^{2} E_{\nu}\left[Z\left(1-4 \sin ^{2} \theta_{\mathrm{W}}\right)-N\right]^{2} \\
& \times\left(E_{\nu}^{\prime}+\left|\mathbf{p}^{\prime}\right| \cos \theta\right),
\end{aligned}
$$

where $Z$ is the nuclear charge and $N$ the neutron number. Henceforth we will neglect the neutral-current term on protons proportional to $\left(1-4 \sin ^{2} \theta_{\mathrm{W}}\right)=0.075$. Performing the final phase-space integral to remove the neutrino scattering angle we find

$\frac{d \sigma_{\mathrm{b}}}{d \omega}=\frac{G_{\mathrm{F}}^{2} N^{2} Z^{2} \alpha}{6 \pi^{2} M^{2} \omega}\left|\mathbf{p}^{\prime}\right|\left[E_{\nu}^{\prime}\left(|\mathbf{p}|^{2}+\left|\mathbf{p}^{\prime}\right|^{2}\right)-\frac{2\left|\mathbf{p} \| \mathbf{p}^{\prime}\right|^{2}}{3}\right]$,

where we may use $|\mathbf{p}|=E_{\nu}$ everywhere.

We will be interested in the endpoint of the cross section, i.e., when $\left|\mathbf{p}^{\prime}\right| \ll|\mathbf{p}|$. In this case we get

$$
\frac{d \sigma_{\mathrm{b}}}{d \omega}=\frac{G_{\mathrm{F}}^{2} N^{2} Z^{2} \alpha}{6 \pi^{2} M^{2} \omega} E_{\nu}^{2} E_{\nu}^{\prime}\left|\mathbf{p}^{\prime}\right|,
$$




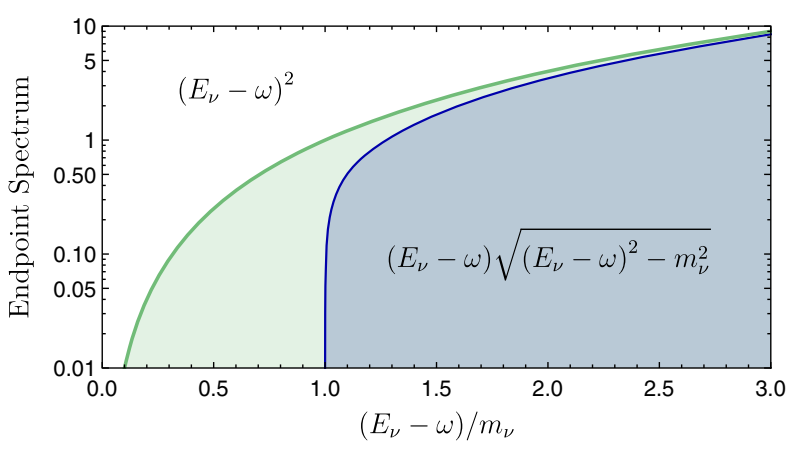

FIG. 2. Endpoint spectrum of $\nu+\mathcal{N} \rightarrow \mathcal{N}+\nu+\gamma$ for monochromatic incident neutrinos (energy $E_{\nu}$ ) with mass $m_{\nu}$ (blue) and zero mass (green). There is no difference between Majorana and Dirac neutrinos when $E_{\nu} \gg m_{\nu} . E_{\nu}-\omega$ is the energy of the outgoing neutrino.

where

$$
E_{\nu}^{\prime}\left|\mathbf{p}^{\prime}\right|=\left(E_{\nu}-\omega\right) \sqrt{\left(E_{\nu}-\omega\right)^{2}-m_{\nu}^{2}}
$$

This result reveals how one would measure the neutrino mass. When the outgoing neutrino is relativistic the cross section scales as $\left(E_{\nu}-\omega\right)^{2}$, but experiences a sharp cutoff at $m_{\nu}$. To see the endpoint explicitly, we plot both $\left(E_{\nu}-\omega\right)^{2}$ and $\left(E_{\nu}-\omega\right) \sqrt{\left(E_{\nu}-\omega\right)^{2}-m_{\nu}^{2}}$ in Fig. 2. As expected, for $\left(E_{\nu}-\omega\right) \lesssim$ few $m_{\nu}$ there is a significant difference between the massive and massless cases.

So far we have considered only a single generation of neutrinos, but all three mass eigenstates are produced by a neutrino source which, if produced by charged-current interactions, provides ratios determined by the mixing angles. As coherent scattering is via the neutral current, all three mass states interact equally. Thus one would in fact see three "endpoints," each causing a dip proportional to the production rate.

\section{DIRAC VS MAJORANA NEUTRINOS}

A longstanding question is whether neutrinos are Dirac or Majorana. The difficulty in answering this question is due to the "Dirac-Majorana confusion theorem." When the neutrino masses are small compared to the other energy scales in the experiment then the differences between Dirac and Majorana neutrino interactions become suppressed by factors of $m_{\nu} / E_{\nu}$ [1]. Specifically, the two Majorana helicity states are equivalent to the left-handed particle and right-handed antiparticle Dirac states when $m_{\nu} \rightarrow 0$. In Ref. [1] the authors noted that the confusion theorem holds even when one of the neutrinos is nonrelativistic; however the relative size of the suppression compared to the purely relativistic case was not studied.

At tree level, for experiments studying tritium or other beta decays [2], there is no difference as the process is via the charged-current interaction. In this case the Feynman rules and thus matrix elements are the same [7]. However, in our neutral-current process there is a difference between the Dirac and Majorana squared matrix elements which is found in the Appendix to be

$$
\Delta\left|\mathcal{M}_{\mathrm{s}}\right|^{2} \sim 4 G_{\mathrm{F}}^{2} M^{2} N^{2} m_{\nu}^{2} .
$$

For simplicity we have neglected here terms that are higher order in $m_{\nu}$ for slow final-state neutrinos as discussed in the Appendix. The fractional difference is

$$
\frac{\Delta\left|\mathcal{M}_{\mathrm{s}}\right|^{2}}{\left|\mathcal{M}_{\mathrm{s}}\right|^{2}} \sim \frac{m_{\nu}^{2}}{E_{\nu}\left(E_{\nu}^{\prime}+\left|\mathbf{p}^{\prime}\right| \cos \theta\right)}
$$

When the final neutrino is nonrelativistic, $\mathrm{E}_{\nu}^{\prime} \sim m_{\nu}$, the fractional difference is $\mathcal{O}\left(m_{\nu} / E_{\nu}\right)$. While for relativistic neutrinos this is $\mathcal{O}\left(m_{\nu}^{2} / E_{\nu}^{2}\right)$, and in this sense our slow finalstate neutrinos somewhat improve the situation, the relative difference is still extremely small. In other words, it is relatively easier to distinguish Dirac and Majorana neutrinos only because the terms which are the same for both Dirac and Majorana neutrinos are suppressed near the kinematic endpoint.

To illustrate the difference, in Fig. 3 we plot the differential cross section $d \sigma / d \omega$ for an exaggerated case of $E_{\nu}=3 m_{\nu}$. To emphasize the behavior near the endpoint we have divided the predicted spectra by $\left|\mathbf{p}^{\prime}\right|$, the momentum of the outgoing neutrino. We assumed a mixture of neutrino helicities as would be produced from a charged current source; however this is not mandatory. Although there are quantitative effects, it seems that there is little characteristic difference between the Majorana and Dirac cases. Such an experiment would require a very strong, low-energy, source of neutrinos.

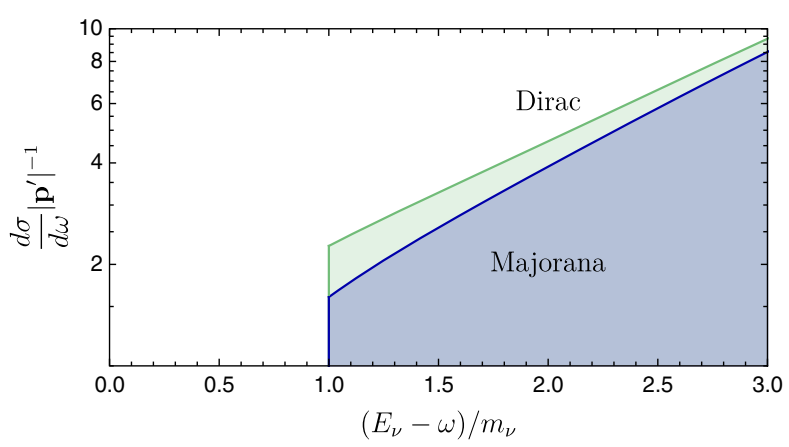

FIG. 3. Differential cross section $d \sigma / d \omega$ vs the scaled final neutrino energy $\left(E_{\nu}-\omega\right) / m_{\nu}$ for Dirac (green) and Majorana (blue) neutrinos. We show a moderately relativistic initial neutrino energy $E_{\nu}=3 m_{\nu}$. We scale by the neutrino momentum $\left|\mathbf{p}^{\prime}\right|$ to better show the fractional differences. 


\section{EXPERIMENTAL ESTIMATES}

\section{A. Endpoint cross section}

Of course, the Dirac vs Majorana question only makes sense if the bremsstrahlung method allows us to find the neutrino mass in the first place. To this end, the photon spectrum needs to be measured with sufficient precision in the endpoint region. A minimal requirement is to measure a significant number of events in a region within a few $m_{\nu}$ of the massless endpoint. To arrive at a numerical estimate we therefore define the endpoint cross section as the integral of the massless cross section from the endpoint to $2 m_{\nu}$,

$$
\begin{aligned}
\sigma_{\text {end }} & =\left.\int_{E_{\nu}-2 m_{\nu}}^{E_{\nu}} \frac{d \sigma_{\mathrm{b}}}{d \omega}\right|_{m_{\nu}=0} d \omega \\
& =\frac{4 \alpha}{9 \pi^{2}}\left(\frac{N Z}{A}\right)^{2} \frac{G_{\mathrm{F}}^{2} E_{\nu} m_{\nu}^{3}}{m_{u}^{2}}+\mathcal{O}\left(m_{\nu}^{4}\right),
\end{aligned}
$$

where $A$ is the mass number of the nucleus, so $M=A m_{u}$ with $m_{u}=930 \mathrm{MeV}$ the atomic mass unit.

We see from Eq. (9) that by going to heavier elements we gain by a coherence factor $(N Z / A)^{2}$. In fact, only the $\nu \mathcal{N}$ scattering process provides a significant coherent enhancement because the bremsstrahlung modification contributes $(Z / A)^{2}$, where the factor $Z^{2}$ comes from the coherent photon interaction with the nucleus and $1 / A^{2}$ from the decrease of $|\Delta \mathbf{p}|^{2} / M^{2}$ for a heavier nucleus.

\section{B. Nuclear recoil approximation}

Our most significant approximation is the neglect of the kinetic energy of the nucleus. This enters in deriving Eq. (2) and the above simplifications of the three-body kinematics. Negligible nuclear recoil is also required experimentally as one needs to avoid excessive blurring of the endpoint spectrum by nucleus recoil. The photon could emerge in any direction relative to the impinging neutrino, so the nucleus needs to absorb a momentum between 0 (forward emitted photon) and $|\Delta \mathbf{q}|=2 E_{\nu}$ (backward emission), so the recoil energy $E_{\text {rec }}$ is in the range between 0 and $\left(2 E_{\nu}\right)^{2} / 2 M=2 E_{\nu}^{2} / M$. The condition $E_{\text {rec }}<m_{\nu}$ implies

$$
E_{\nu}<\sqrt{2 M m_{\nu}}=590 \mathrm{keV} \sqrt{\frac{A}{184} \frac{m_{\nu}}{1 \mathrm{eV}}},
$$

where $m_{\nu}$ is the smallest neutrino mass to which the experiment could be sensitive and the numerical value is for tungsten with an atomic weight of $A=184$. When this approximation is not valid more detailed calculations are necessary.

\section{Estimated cross section}

We use tungsten $(Z=74)$ as an example as used in the coherent scattering project " $\nu$-cleus" [8]. Using the largest useful $E_{\nu}$ of Eq. (10) as a benchmark, our endpoint cross section on tungsten is

$$
\sigma_{\text {end }}=2.3 \times 10^{-68} \mathrm{~cm}^{2}\left(\frac{m_{\nu}}{1 \mathrm{eV}}\right)^{3.5} .
$$

Evidently a very powerful source of monochromatic neutrinos would be needed to carry out such a study experimentally.

The most promising source would consist of elements which decay solely through electron capture. Two possible candidates are ${ }^{68} \mathrm{Ge}$ and ${ }^{51} \mathrm{Cr}$. Both decay via electron capture, with a lifetime of 270 and 27 days respectively. Chromium was planned as the source for the SOX experiment [9] and was used in the GALLEX experiment [10]. To study the Majorana vs Dirac question the neutrino source would additionally have to be of low energy so the $m_{\nu} / E_{\nu}$ is not entirely negligible. In that case, Ge with its decay at $110 \mathrm{keV}$ would be preferable, and would allow one to probe $m_{\nu} \sim 0.1 \mathrm{eV}$.

Either way, it would be extremely challenging to find a combination of realistic source power and detector size capable of overcoming the small cross section of Eq. (11). While experiments using these types of sources have been performed, the relevant cross section was an ordinary weak one. In particular for the GALLEX experiment using a ${ }^{51} \mathrm{Cr}$ source, the absorption cross section on gallium was $58 \times 10^{-46} \mathrm{~cm}^{2}$ [10]. In our case, we need many events in the very narrow energy range near the endpoint defined by the neutrino search mass, which is what makes Eq. (11) so small. Moreover, the nature of our process as a radiative process of the order of $\alpha$ relative to a weak process and the nonrelativistic nature of the radiation process, bringing in the factor $|\Delta \mathbf{p}|^{2} / M^{2} \ll 1$, is not completely overcome by coherence factors.

One possible way to improve the cross section would be to consider an electron rather than a nucleus as a target to avoid the large $1 / M^{2}$ factor. Unfortunately one loses the coherent enhancement of the cross section, and must deal with the significantly more complicated kinematics. A similar process was considered in [11], though not for the purposes of neutrino mass detection.

\section{CONCLUSIONS}

In this paper we have explored the possibility of using coherently enhanced inelastic neutrino scattering to determine the neutrino masses. As the photon can carry the entire available kinetic energy of the system one could measure the masses of the neutrinos by measuring the endpoint of the photon spectrum.

To this end, we calculated the coherent bremsstrahlung produced by neutrino scattering for both Dirac and Majorana neutrinos. We showed that suppressions to the rate caused by the low velocity of the nucleus and by moving to the kinematic endpoint strongly reduce the cross section relative to an ordinary weak one.

Although impractically intense monochromatic neutrino beams would seem to be needed for an experiment, a 
number of interesting points arise from the calculation. Since we go beyond the soft photon approximation we are able to establish the shape of the photon spectrum near the endpoint, namely, $E_{\nu}^{\prime}\left|\mathbf{p}^{\prime}\right|=\left(E_{\nu}-\omega\right) \sqrt{\left(E_{\nu}-\omega\right)^{2}-m_{\nu}^{2}}$.

Further, we explored the confusion theorem in this context. While in principle there is a difference in the rate between Dirac and Majorana neutrinos, it is suppressed by a factor of $m_{\nu} / E_{\nu}$ and so one would need an exceedingly precise measurement with a low-energy source to detect it.

Finally we would like to note the principle we apply here could be applied to other reactions, some of which might be more favorable experimentally. These processes would consist of a quasielastic neutrino scattering, leading to a three-body final state. Taking one of the particles to its kinematic endpoint can lead to a "stopping" final neutrino.

\section{ACKNOWLEDGMENTS}

We acknowledge partial support by the Deutsche Forschungsgemeinschaft through Grants No. EXC 153 (Excellence Cluster Universe) and No. SFB 1258 (Collaborative Research Center "Neutrinos, Dark Matter, Messengers") as well as by the European Union through Grants No. H2020-MSCA-ITN-2015/674896 (Innovative Training Network "Elusives") and No. H2020-MSCARISE-2015/690575 (Research and Innovation Staff Exchange project "Invisibles Plus"). A. M. is supported by the European Research Council under Grant No. 742104.

\section{APPENDIX: MATRIX ELEMENTS}

We calculate the matrix element for the scattering process $\nu+\mathcal{N} \rightarrow \mathcal{N}+\nu$ of a relativistic Dirac or Majorana neutrino with mass $m_{\nu}$ and energy $E_{\nu} \gg m_{\nu}$ on a heavy nucleus with mass $M \gg E_{\nu}$. Using the Feynman rules of Ref. [7] we find

$$
\begin{aligned}
& \mathcal{M}_{\mathrm{s}}^{\mathrm{D}}=\frac{G_{\mathrm{F}}}{2 \sqrt{2}} \bar{\nu}_{\mathrm{f}} \gamma^{\mu}\left(1-\gamma^{5}\right) \nu_{\mathrm{i}} J_{\mu}^{\mathcal{N}}, \quad \text { Dirac } \\
& \mathcal{M}_{\mathrm{s}}^{\mathrm{M}}=\frac{G_{\mathrm{F}}}{\sqrt{2}} \bar{\nu}_{\mathrm{f}} \gamma^{\mu} \gamma^{5} \nu_{\mathrm{i}} J_{\mu}^{\mathcal{N}}, \quad \text { Majorana }
\end{aligned}
$$

where we have neglected global phases. Moreover

$$
J_{\mu}^{\mathcal{N}}=\left[\left(1-4 \sin ^{2} \theta_{\mathrm{w}}\right) Z-N\right]\left(q+q^{\prime}\right)_{\mu}
$$

is the matrix element of the nucleus current with $N$ the number of neutrons and $Z$ that of protons. We have assumed the nucleus to be scalar or that it has so many nucleons that we can ignore the axial interaction relative to the coherent vector one. Note that for the Majorana case we simply needed to replace $\left(1-\gamma^{5}\right)$ with $2 \gamma^{5}$; i.e., a Majorana neutrino only couples axially.

One can actually see from this observation that the confusion theorem must apply even when only one of the neutrinos is relativistic [1]. For the Dirac case, to get to Eq. (A1) we must evaluate the spinor expression $\bar{u}\left(p^{\prime}\right) \gamma_{\mu}\left(1-\gamma_{5}\right) u(p)$. For the Majorana case there is only the axial current, $2 \bar{u}\left(p^{\prime}\right)\left(\gamma_{\mu} \gamma_{5}\right) u(p)$, to evaluate. The two cases will be equivalent only if the vector current term yields the same as the axial current term. This will be the case if one of the $u$ 's is an eigenstate of $\gamma_{5}$, that is, a helicity state. In particular, only one of the $u$ 's needs to be a helicity state since the $\gamma_{5}$ can be passed from one side to the other with merely a sign change. Thus in the relativistic limit the difference between Dirac and Majorana will always be suppressed. To see the form more explicitly, however, we must preform the full calculation.

We consider the nucleus to be highly nonrelativistic and recoil effects can be neglected, implying that we can use $J^{\mathcal{N}}=-N(2 M, \mathbf{0})$, where we neglect the contribution from protons as explained in the main text. Thus we only need the 0 component of the neutrino current. Since we will eventually consider the case $E_{\nu} \gg m_{\nu}$ the initial neutrino source will have an almost definite helicity. In the squared matrix element we find, assuming an initial helicity $h$ and summing over the final one,

$$
\begin{aligned}
\left|\bar{\nu}_{\mathrm{f}} \gamma^{0}\left(1-\gamma^{5}\right) \nu_{\mathrm{i}}\right|^{2} & =8\left(E_{\nu}-h|\mathbf{p}|\right)\left(E_{\nu}^{\prime}-h\left|\mathbf{p}^{\prime}\right| \cos \theta\right), \\
\left|2 \bar{\nu}_{\mathrm{f}} \gamma^{0} \gamma^{5} \nu_{\mathrm{i}}\right|^{2} & =16\left(E_{\nu} E_{\nu}^{\prime}+|\mathbf{p}|\left|\mathbf{p}^{\prime}\right| \cos \theta-m_{\nu}^{2}\right),
\end{aligned}
$$

where $\theta$ is the neutrino scattering angle, $E_{\nu}=\sqrt{\mathbf{p}^{2}+m_{\nu}^{2}}$ and analogous for $E_{\nu}^{\prime}$. To write the above we used [12]

$$
\nu \bar{\nu}=\frac{1}{2}\left(\not p+m_{\nu}\right)\left(1+h \gamma^{5} \not\right)
$$

with the spin vector

$$
S=\left(\frac{|\mathbf{p}|}{m_{\nu}}, \frac{E_{\nu}}{m_{\nu}} \hat{\mathbf{p}}\right) .
$$

Note that in the Majorana case the result of Eq. (A3b) does not depend on the initial helicity $h$. This is because in the relativistic limit the two helicities play the role of neutrino and antineutrino and the vector interaction rate of the neutral current is the same for $\nu$ and $\bar{\nu}$. Including axialcurrent interactions with nuclei, the $\nu$ and $\bar{\nu}$ scattering rates would be different due to weak magnetism [13]. In the Dirac case, for $h=-1$, the result is the same as for Majorana neutrinos up to $m_{\nu}^{2}$ corrections, whereas for $h=+1$ it is much smaller and vanishes for $m_{\nu}=0$ because this "wrong-helicity" case represents the sterile righthanded Dirac component.

For nonvanishing $m_{\nu}$, neutrinos from the source have a "wrong-helicity" component with a probability $\left(m_{\nu} / 2 E_{\nu}\right)^{2}$, which should be included in the rate. For a nice demonstration of this point, see Appendix A of Ref. [14]. Thus there are in principle three effects that can distinguish 
between Dirac and Majorana neutrinos: (i) the higher probability of $h=+1$ Majorana neutrinos to interact; (ii) the difference between $E_{\nu}$ and $|\mathbf{p}|$, which enters via different $S$ dependence of Dirac and Majorana neutrinos; and (iii) the extra term $m_{\nu}^{2}$ in the Majorana case which tends to cancel the energy terms for small energies. In the elastic case, this term decouples the neutrino from the neutral current at low energies.

Putting this all together with $1-\left(m_{\nu} / 2 E_{\nu}\right)^{2}$ as the probability for the $h=-1$ initial state and $\left(m_{\nu} / 2 E_{\nu}\right)^{2}$ for the $h=+1$ one, we find for the summed squared matrix elements and the modified flux factors

$$
\begin{aligned}
\left|\mathcal{M}_{\mathrm{s}}^{\mathrm{D}}\right|^{2}= & 2 G_{\mathrm{F}}^{2} N^{2} M^{2}\left(1-\frac{m_{\nu}^{2}}{4 E_{\nu}^{2}}\right) \\
& \times\left(E_{\nu}+|\mathbf{p}|\right)\left(E_{\nu}^{\prime}+\left|\mathbf{p}^{\prime}\right| \cos \theta\right),
\end{aligned}
$$

$\left|\mathcal{M}_{\mathrm{s}}^{\mathrm{M}}\right|^{2}=4 G_{\mathrm{F}}^{2} N^{2} M^{2}\left(E_{\nu} E_{\nu}^{\prime}+\left|\mathbf{p} \| \mathbf{p}^{\prime}\right| \cos \theta-m_{\nu}^{2}\right)$.

In the Dirac case the correction due to the $h=+1$ component is of order $\left(m_{\nu} / 2 E_{\nu}\right)^{4}$ through the flux factor $\left(m_{\nu} / 2 E_{\nu}\right)^{2}$ and the cancellation in $(E-|\mathbf{p}|)$, and it has been left out. Thus these relations are exact up to $\mathcal{O}\left(m_{\nu}^{2}\right)$. We will consider the case where $E_{\nu}^{\prime} \ll E_{\nu}$ so we can simplify these expressions by neglecting the wrong-helicity neutrinos and setting $E_{\nu}=|\mathbf{p}|$ and we find

$\left|\mathcal{M}_{\mathrm{s}}^{\mathrm{D}}\right|^{2}=4 G_{\mathrm{F}}^{2} N^{2} M^{2}\left(E_{\nu} E_{\nu}^{\prime}+E_{\nu}\left|\mathbf{p}^{\prime}\right| \cos \theta\right)$,

$\left|\mathcal{M}_{\mathrm{s}}^{\mathrm{M}}\right|^{2}=4 G_{\mathrm{F}}^{2} N^{2} M^{2}\left(E_{\nu} E_{\nu}^{\prime}+E_{\nu}\left|\mathbf{p}^{\prime}\right| \cos \theta-m_{\nu}^{2}\right)$.

These are the expressions that are relevant for the endpoint spectrum discussed in the main text.
[1] B. Kayser, F. Gibrat-Debu, and F. Perrier, The physics of massive neutrinos, World Sci. Lect. Notes Phys. 25, 1 (1989).

[2] J. Wolf et al. (KATRIN Collaboration), The KATRIN neutrino mass experiment, Nucl. Instrum. Methods Phys. Res., Sect. A 623, 442 (2010).

[3] C. Kouvaris and J. Pradler, Probing Sub-GeV Dark Matter with Conventional Detectors, Phys. Rev. Lett. 118, 031803 (2017).

[4] D. Akimov et al. (COHERENT Collaboration), Observation of coherent elastic neutrino-nucleus scattering, Science 357, 1123 (2017).

[5] J. M. Berryman, A. de Gouva, K. J. Kelly, and M. Schmitt, Shining light on the mass scale and nature of neutrinos with

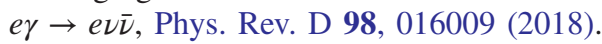

[6] A. Drukier and L. Stodolsky, Principles and applications of a neutral-current detector for neutrino physics and astronomy, Phys. Rev. D 30, 2295 (1984).

[7] J. Gluza and M. Zralek, Feynman rules for Majorana neutrino interactions, Phys. Rev. D 45, 1693 (1992).

[8] R. Strauss et al., The $\nu$-cleus experiment: A gram-scale fiducial-volume cryogenic detector for the first detection of coherent neutrino-nucleus scattering, Eur. Phys. J. C 77, 506 (2017).

[9] G. Bellini et al. (Borexino Collaboration), SOX: Short distance neutrino oscillations with BoreXino, J. High Energy Phys. 08 (2013) 038.

[10] W. Hampel et al. (GALLEX Collaboration), Final results of the ${ }^{51} \mathrm{Cr}$ neutrino source experiments in GALLEX, Phys. Lett. B 420, 114 (1998).

[11] E. Akhmedov, G. Arcadi, M. Lindner, and S. Vogl, Coherent scattering and macroscopic coherence: Implications for neutrino, dark matter and axion detection, J. High Energy Phys. 10 (2018) 045.

[12] A. J. Long, C. Lunardini, and E. Sabancilar, Detecting non-relativistic cosmic neutrinos by capture on tritium: Phenomenology and physics potential, J. Cosmol. Astropart. Phys. 08 (2014) 038.

[13] C. J. Horowitz, Weak magnetism for antineutrinos in supernovae, Phys. Rev. D 65, 043001 (2002).

[14] P. Langacker and J. Wang, Neutrino anti-neutrino transitions, Phys. Rev. D 58, 093004 (1998). 